\title{
Correction to: The Pharmacology and Clinical Efficacy of Antiseizure Medications: From Bromide Salts to Cenobamate and Beyond
}

\author{
Wolfgang Löscher ${ }^{1,2}$ (D) $\cdot$ Pavel Klein ${ }^{3}$
}

Published online: 17 August 2021

(c) Springer Nature Switzerland AG 2021

\section{Correction to: CNS Drugs \\ https://doi.org/10.1007/s40263-021-00827-8}

In the original publication, the following errors were identified in Fig. 1:

1. The structure of felbamate was shown incorrectly (instead of felbamate, cenobamate was shown).

2. The structure of brivaracetam was shown incorrectly (a hydroxyl group was shown instead of a keto group).

In addition, the order of brivaracetam and levetiracetam was incorrect (levetiracetam was developed earlier).

The corrected Fig. 1 is given below.

The original article has been corrected.

The original articles can be found online at https://doi.org/10. 1007/s40263-021-00827-8.

Wolfgang Löscher

wolfgang.loescher@tiho-hannover.de

1 Department of Pharmacology, Toxicology, and Pharmacy, University of Veterinary Medicine, Bünteweg 17,

30559 Hannover, Germany

2 Center for Systems Neuroscience, Hannover, Germany

3 Mid-Atlantic Epilepsy and Sleep Center, Bethesda, MD, USA 

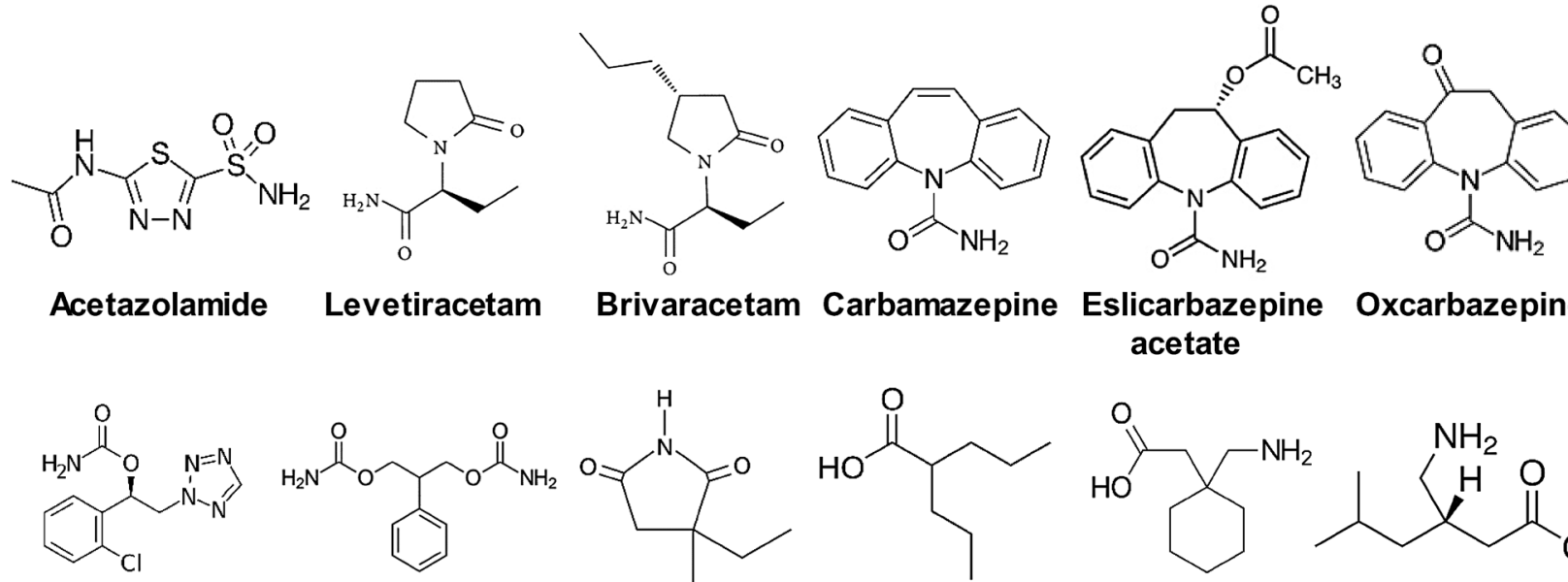<smiles>CCC1(C)CC(=O)NC1=O</smiles><smiles>CCCC(CCC)C(=O)O</smiles><smiles>NCC1(CC(=O)O)CCCCC1</smiles><smiles>CC(C)C[C@H](CN)CC(=O)O</smiles>

\section{Cenobamate}

Felbamate

Ethosuximide Valproic acid

\section{Gabapentin}

\section{Pregabalin}<smiles></smiles><smiles>C=CC(N)CCC(=O)O</smiles><smiles>COCC(NC(C)=O)C(=O)NCc1ccccc1</smiles><smiles>CN1C(=O)CC(=O)N(c2ccccc2)c2cc(Cl)ccc21</smiles><smiles>N#Cc1ccccc1-c1cc(-c2ccccc2)cn(-c2ccccc2)c1=O</smiles>

Tiagabine

Vigabatrin

\section{Lamotrigine}

Lacosamide

\section{Clobazam}

Perampanel<smiles>CCC1(c2ccccc2)C(=O)NC(=O)NC1=O</smiles><smiles>O=C1NC(=O)C(c2ccccc2)(c2ccccc2)N1</smiles><smiles>O=C1NC(c2ccccc2)(c2ccccc2)C(=O)N1COP(=O)(O)O</smiles><smiles>CCC1(c2ccccc2)C(=O)NCNC1=O</smiles><smiles>NC(=O)c1cn(Cc2c(F)cccc2F)nn1</smiles><smiles>NS(=O)(=O)Cc1noc2ccccc12</smiles>

Phenobarbital

Phenytoin

Fosphenytoin

Primidone

Rufinamide

Zonisamide<smiles>CC(C)C(C)(C)C(C)(C)/C=C/c1ccc2c(c1)OCO2</smiles>

Topiramate
Stiripentol<smiles>NS(=O)(=O)c1ccc(N2CCCCS2(=O)=O)cc1</smiles>

Sulthiame

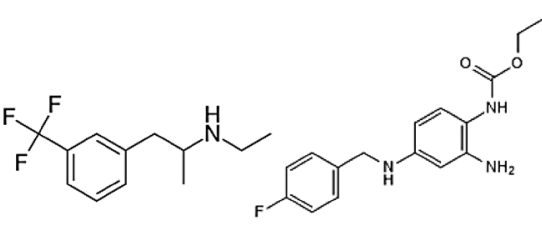

Fenfluramine Retigabine

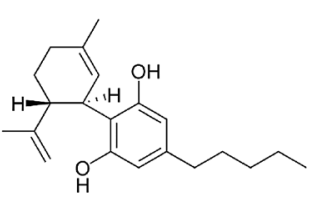

Cannabidiol

Fig. 1 Chemical structures of clinically approved antiseizure drugs discussed in this review 